\title{
Effets climatiques sur les écosystèmes marins Exemple du recrutement des Crustacés Cirripèdes sur la côte ouest du Cotentin
}

\section{Climatic changes on Marine Ecosystems Example of the recruitment of intertidal Barnacles from the west coast of Cotentin}

\section{Luc Drévès}

Ifremer - Centre de Brest

Direction de l'Environnement et de l'Aménagement littoral

Service Régional de l'Environnement

BP 70 - 29280 Plouzané - France

E-mail : luc.dreves@ifremer.fr

Résumé - Le cap de Flamanville, sur la côte ouest du Cotentin (Manche ouest, France), a été choisi par E.D.F. pour l'implantation d'une centrale électronucléaire en bordure du littoral.

Parmi les différents paramètres mesurés au cours de l'étude de projet (1976-1978), la recolonisation des substrats durs intertidaux par les crustacés cirripèdes a été étudiée. Dans un peuplement dominé à $80 \%$ par l'espèce Balanus balanoides, l'importance du recrutement printanier dans le cycle annuel a été mis en évidence.

Cette étude a été poursuivie à partir de 1983, et ceci sans discontinuité jusqu'en 2001 , dans le cadre du programme réglementaire de surveillance de la centrale.

D'importantes variations du recrutement printanier sont constatées d'une année sur l'autre.

Une relation est établie entre la rigueur de l'hiver (température mensuelle moyenne) et l'importance numérique de ces cirripèdes qui se fixent sur le substrat rocheux entre mars et juin.

Mots-clés - Centrale nucléaire, Flamanville, Surveillance; crustacés cirripèdes, Balanus balanoides, recrutement.

Abstract - A nuclear power plant is set up at Flamanville (Channel; west coast of the Cotentin).

For monitoring studies, one species (or a group of species) has been chosen in each major unit for a reference population dynamics study.

Hard bottoms are covered by several species of barnacles. These species present different temperature affinities, and have reached here an equilibrium stage. The recruit- 
ment period of Balanus balanoides, the main species, has been determined. A relationship is established between the wintry temperature and the numerical importance of its spring recruitment.

Keywords - Flamanville, Monitoring, Nuclear power plant ; Balanus balanoides, Barnacles, Recruitment.

Dans le cadre de l'implantation d'un Centre National de Production d'Electricité (CNPE) sur le littoral de la commune de Flamanville (fig. 1; Département de la Manche) et du contexte législatif en vigueur, ELECTRICITÉ DE FRANCE a confié à I'IFREMER les études écologique et halieutique du milieu marin encadrant ce site. Ces travaux ont été réalisés dans le cadre des études d'avant-projet (1975), de projet (1976-1979), et se sont poursuivis dans le cadre des études de surveillance (1983-2002).

Parmi les différents paramètres(") mesurés au cours de l'étude de projet et conservés dans le programme de surveillance, figure l'étude du peuplement animal des substrats durs intertidaux. Sont ainsi étudiées les variations d'abondance des principales espèces de crustacés cirripèdes dans la ceinture à Balanus balanoides, la recolonisation du substrat par ces espèces, et l'évaluation sommaire des densités des principales espèces accompagnatrices appartenant à l'embranchement des mollusques.

(1) Domaine pélagique: hydrobiologie, phytoplancton, zooplancton, microbiologie. Domaine benthique : phyto- et zoobenthos.

- Domaine halieutique: zooplancton, hydrologie, suivi de l'exploitation et de la biologie des especes commerciales.
Les résultats présentés ci-après concernent la recolonisation du substrat par les cirripèdes et leur variation d'abondance, tels que présentés chaque année dans le rapport scientifique remis à EDF (Drévès et al., 2002).

\section{RAPPEL DES CONCLUSIONS DES ÉTUDES DE PROJET (1976-1979)}

Le peuplement des crustacés cirripèdes intertidaux, étudié à la pointe du Rozel de décembre 1977 à juin 1978 (Guillaumont \& Hamon, 1981), présente des densités particulièrement élevées (40000 à 70000 individus par $\mathrm{m}^{2}$ ). Quatre espèces, présentant des affinités thermiques différentes, s'y trouvent en compétition directe pour l'espace (fig. 2) :

- Balanus balanoides est une espèce boréo-arctique qui ne s'étend guère, vers le sud, au-delà des côtes atlantiques (estuaires du nord-ouest de l'Espagne); à la pointe du Rozel, cette espèce domine très largement le peuplement (aux alentours de $94 \%$ );

- Elminius modestus, espèce originaire d'Australie et de NouvelleZélande introduite en Europe vers 


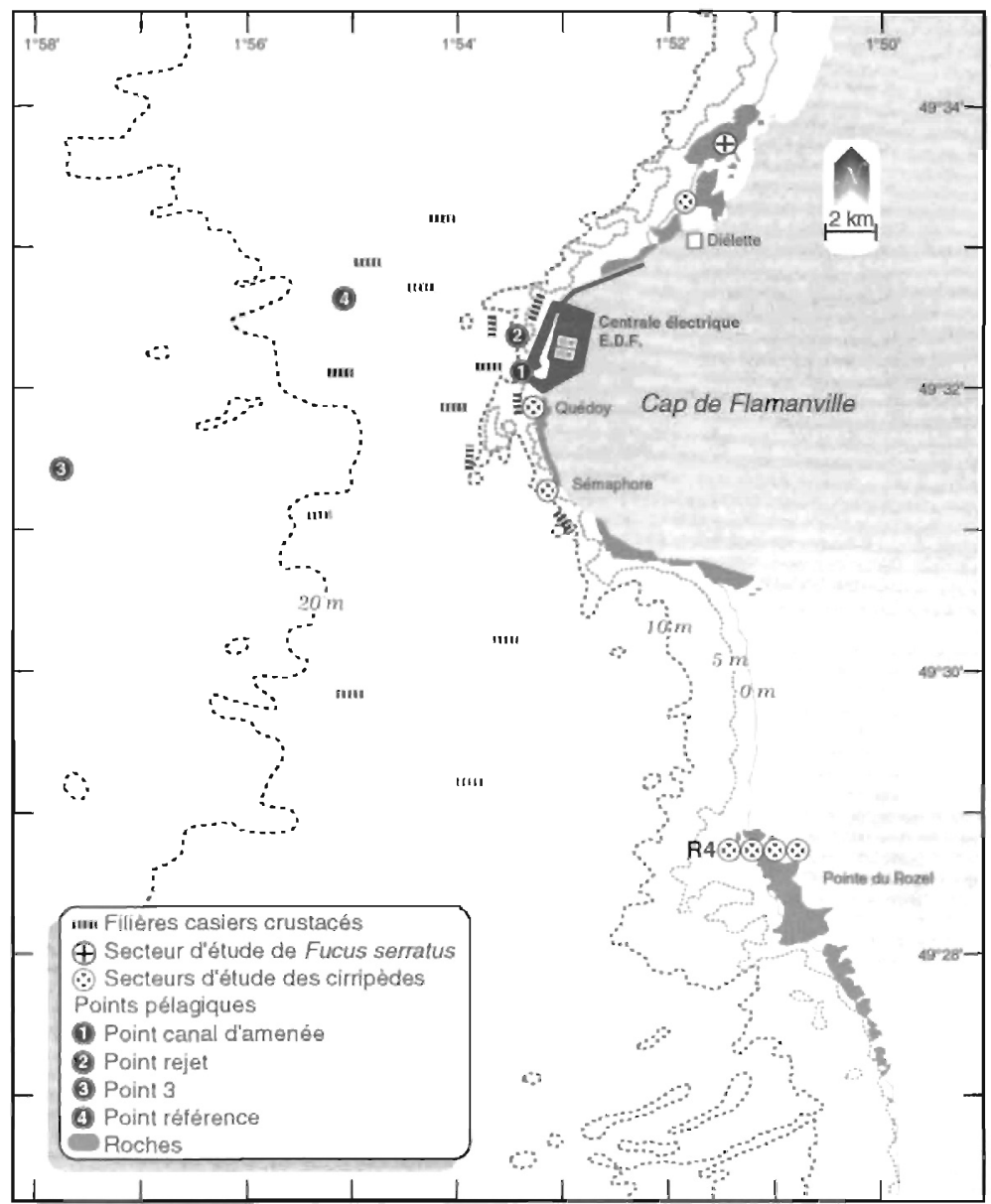

Fig. 1 - Carte des abords de Flamanville indiquant les différents points de prélèvements échantillonnés par lifremer

Fig. 1 - Study area in front of Flamanville. Location of sampling points.

1940, est aujourd'hui recensé du Portugal à l'Irlande et au Danemark; dans le nord-Cotentin, elle représente entre 5 et $6 \%$ du peuplement;

- Chthamalus stellatus est une espèce présente en Méditerranée, ainsi que de l'ouest de l'Afrique à
l'Ecosse ; la presqu'île du Cotentin constitue sa limite nord de répartition pour les côtes françaises;

- Chthamalus montagui, espèce décrite par Southward (1976), est présent du nord de l'Ecosse au Maroc, et semble être absent des côtes méditerranéennes. 


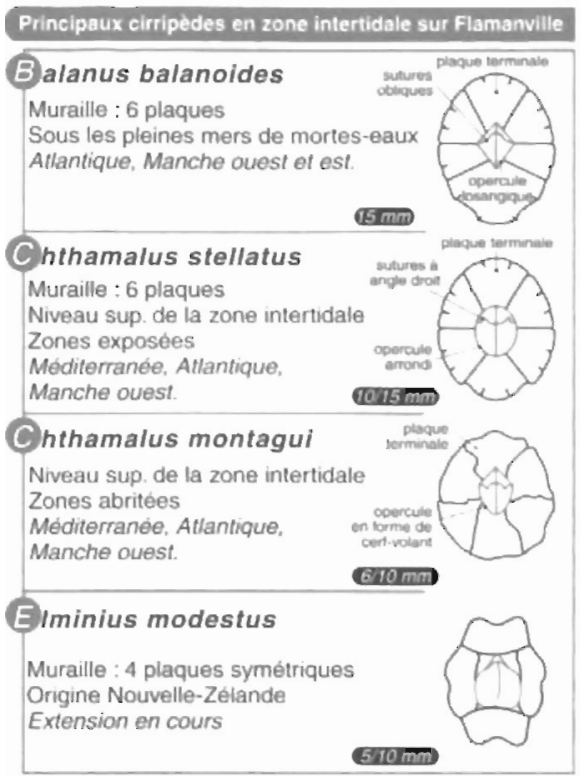

Fig. 2 - Principaux cirripèdes intertidaux sur Flamanville

Fig. 2 - Main intertidal barnacles species in front of Flamanville

La période principale de recrutement s'étend de mars à juin, avec un maximum en mai (fig. 3 ). Ce recrutement printanier, qui représente près de $98 \%$ du phénomène annuel, est presque exclusivement le fait des in-

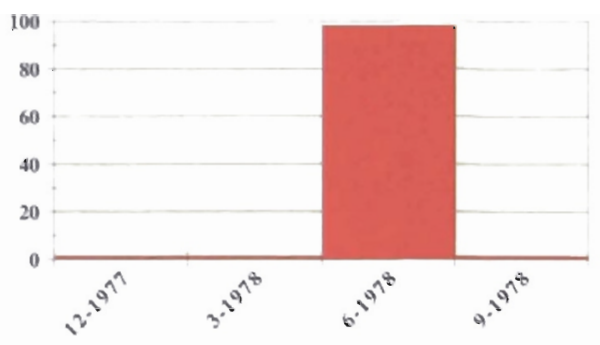

Fig. 3 - Importance, en nombre d'individus fixés par $75 \mathrm{~cm}^{2}$, de la recolonisation du substrat rocheux.

Fig. 3 - Abondance (number of individuals per $75 \mathrm{~cm}^{2}$ ) of the recruitment on the rocky shore.

dividus de Balanus balanoides. Pour l'espèce Elminius modestus, le recrutement est faible et étalé dans le temps. Quant aux Chthamales, ils ne sont représentés sur le site que par de vieux et rares individus, aucun recrutement n'étant observé.

Les pourcentages de Balanus balanoides et Elminius modestus, obtenus en fin de périodes de recolonisation sur des substrats vierges (fig. 4), sont du même ordre que ceux des populations déjà en place. Ceci laisse à penser que ces espèces compétitives sont, sur ce site, en état d'équilibre.

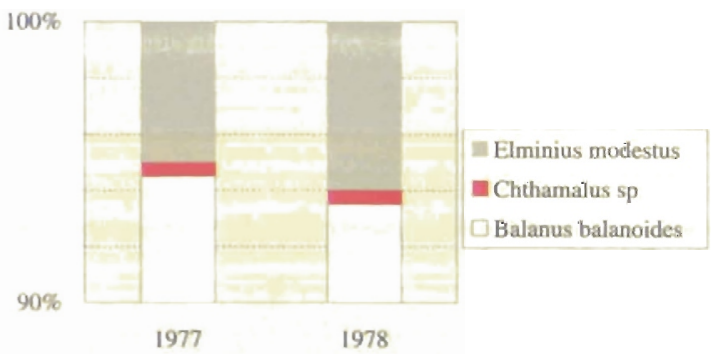

Fig. 4 - Pourcentage des quatre espèces de cirripèdes à la Pointe du Fozel en 1977 et 1978. Les deux espèces de Chthamales ont été regroupées au ni= veau du genre.

Fig. 4 - Proportion of the four intertidal barnacle species in 1977 and 1978 at the Pointe du Rozel 


\section{PROTOCOLE D'ANALYSES DES ÉTUDES DE SURVEILLANCE (1983-2002)}

A la pointe du Rozel (fig. 1), quatre points de la radiale $R 4$ sont échantillonnés dans la ceinture à Balanus balanoides à raison d'un point par strate. Au cap de Flamanville, les trois points "sémaphore" (ou SEM; depuis 1983), "Quédoy" et "Diélette " (à partir de 1993) sont retenus comme représentatifs de l'ensemble de la ceinture à l'exception de la frange supérieure.

En septembre, la densité de cirripèdes est évaluée à l'aide de quadrats $0,05 \mathrm{~m} \times 0,05 \mathrm{~m}$ (8 quadrats par point); le pourcentage de recouvrement du substrat par ces crustacés est noté. L'échantillonnage est stratifié pour tenir compte de l'hétérogénéité de colonisation (zone à forte densité, faible densité, absence totale de cirripèdes), phénomène constaté essentiellement aux limites de la ceinture. Des échantillons de substrat sont rapportés au laboratoire pour l'évaluation des pourcentages des différentes espèces.

Les points Rozel ( $\left.n^{\circ} 2\right)$, SEM, Quédoy et Diélette servent également à une étude de recolonisation du substrat par les cirripèdes. Dans ce but, des comptages puis des grattages sont réalisés sur les mêmes séries de quadrats $\left(75 \mathrm{~cm}^{2}\right)$ à trois périodes de l'année, en fin d'hiver (mars - avril), fin de printemps (juin) et fin d'été (septembre). Ceci permet de cerner l'importance de la fixation des recrues selon les saisons et d'établir le bilan global de recolonisation annuelle. Afin de limiter l'invasion des surfaces mises à nu par les espèces d'épifaune vagile (patelles, littorines) les quadrats rectangulaires sont de faible largeur $(3 \mathrm{~cm} \times 25 \mathrm{~cm})$. La disposition des quatre quadrats est représentée dans le schéma (fig. 5).

La recolonisation notée lors d'une mission donnée est le résultat du recrutement saisonnier en cours (incubation des œufs à l'abri de la muraille, éclosion et libération des larves nageuses de type nauplius dans la masse d'eau), diminué d'une mortalité (non quantifiée) intervenue entre la date de fixation des cypris (larves mé-

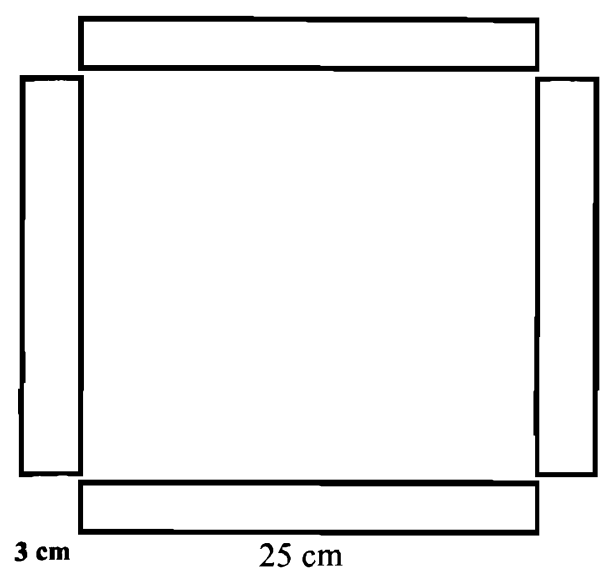

Fig. 5 - Disposition des quatre quadrats de faible largeur pour l'étude de colonisation du substrat rocheux.

Fig. 5 - On the rocky shore every year, in March, 4 quadrats $(3 \mathrm{~cm} \times 25 \mathrm{~cm})$ are scraped off. In June, census of barnacles, providing an estimate of the individuals recruited in spring; then the same 4 quadrats are again scraped off. In September, census and estimation of the density of summer recruits, then scraping off. In March, autumn-winter estimate... etc. 
tamorphosées) sur le substrat et la date d'observation.

\section{PRINCIPAUX RÉSULTATS DES ÉTUDES DE SURVEILLANCE}

\section{Densités et pourcentages des différentes espèces de cirripèdes}

Les densités de cirripèdes, observées en fin d'été (fig. 6), montrent des variations pluriannuelles importantes en tout point, révélatrices de la variabilité naturelle.

Ces variations découlent de la surface de substrat rocheux colonisé d'une part (de $40 \%$ à $100 \%$ ), de l'importance du recrutement, surtout printanier, d'autre part. Ces fluctuations affectent principalement l'espèce dominante, Balanus balanoides.

Sur la période 1983-2001, les densités globales varient de 92000 individus par $\mathrm{m}^{2}$ en 1987 à 18000 individus par $\mathrm{m}^{2}$ en 1990. Ces valeurs extrêmes, notées au Rozel, encadrent bien celles enregistrées au cours des études de projet (40000 à 70000 individus par $\mathrm{m}^{2}$ ). En contre-bas du sémaphore, les variations sont d'ampleurs sensiblement moindres (de 91000 à 23000 individus par $\mathrm{m}^{2}$ ).

En tout point, les densités de Chthamalus sp sont faibles, la moyenne sur la période d'observations étant de 600 individus par $\mathrm{m}^{2}$ au Rozel, 900 à Diélette, 5500 au sémaphore et 9200 à Quédoy. Dans un contexte de décroíssance des effectifs globaux de cirripèdes en ces points, le constat est fait du maintien de densités de Chthamalus sp plus élevées au sémaphore et à Quédoy, point où une augmentation sensible de leurs effectifs serait perceptible (observation mentionnée avec prudence au regard de la courte durée [ 8 ans] de la série chronologique).
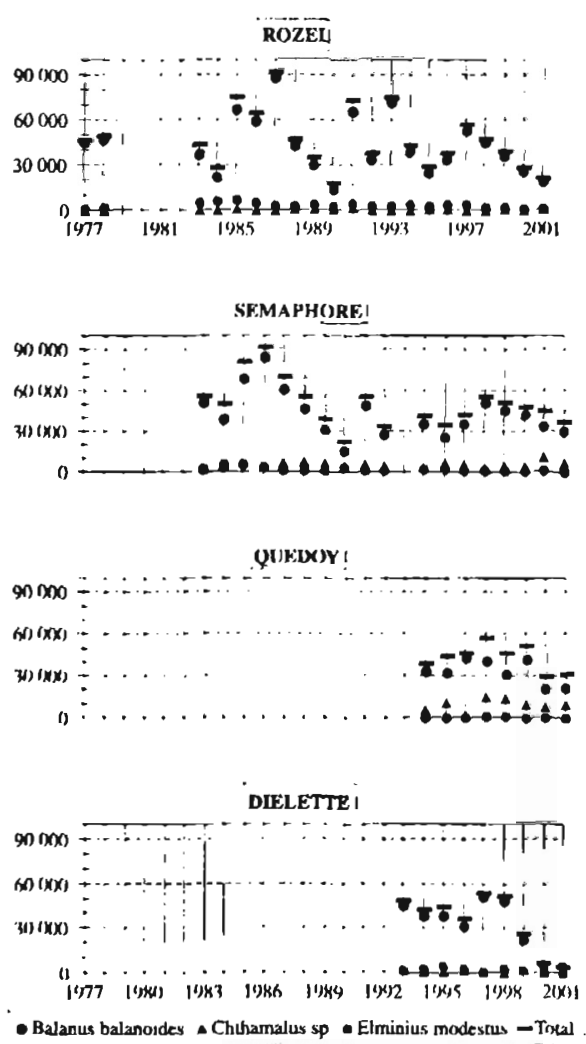

Fig.6 - Evolution, en nombre d'individus par $m^{\prime}$, des densités des quatre espèces de cirripèdes.

Fig. 6 - Long-term variation in population densities (N.ind. $\mathrm{m}^{2}$ ) of four intertidal barnacle species. 
Ces deux stations se distinguent des deux autres par leur proximité de la centrale et par la nature granitique du substrat.

Les proportions de ces quatre espèces de crustacés cirripèdes sont calculées en chaque point. Comme pour les densités, les variations observées (fig. 7) se répercutent principalement sur l'espèce dominante Balanus balanoides.

Appréciés aux alentours de $94 \%$ à la pointe du Rozel lors des études de projet, les pourcentages de cette espèce y varient de $78 \%$ (années 1984 et 1990 ) à $96 \%$ (1987 et 1996).

Au cap de Flamanville, des pourcentages plus faibles sont enregistrés: $68 \%$ en 1990 au sémaphore, $69 \%$ en 2001 à Quédoy. Ce dernier point, suivi seulement depuis 1994 , est celui où les Balanus balanoides sont le moins bien représentés.

Inversement, les pourcentages de Chthamales y sont les plus forts, progressant de façon très irrégulière $(6 \%$ en 1996) de $13 \%$ en 1994 à près de $31 \%$ en 2001.

Au sémaphore, les Chthamales sont également bien représentés. Leurs pourcentages passent, d'une manière très irrégulière, de $3 \%$ en 1983 à $24 \%$ en 2000.

A la pointe du Rozel, les Chthamales ne représentaient que 0,2 à $0,3 \%$ du peuplement en 1977 et 1978. Depuis 1983, leur présence demeure faible, le maximum observé n'étant que de $3,8 \%$ en 1990.
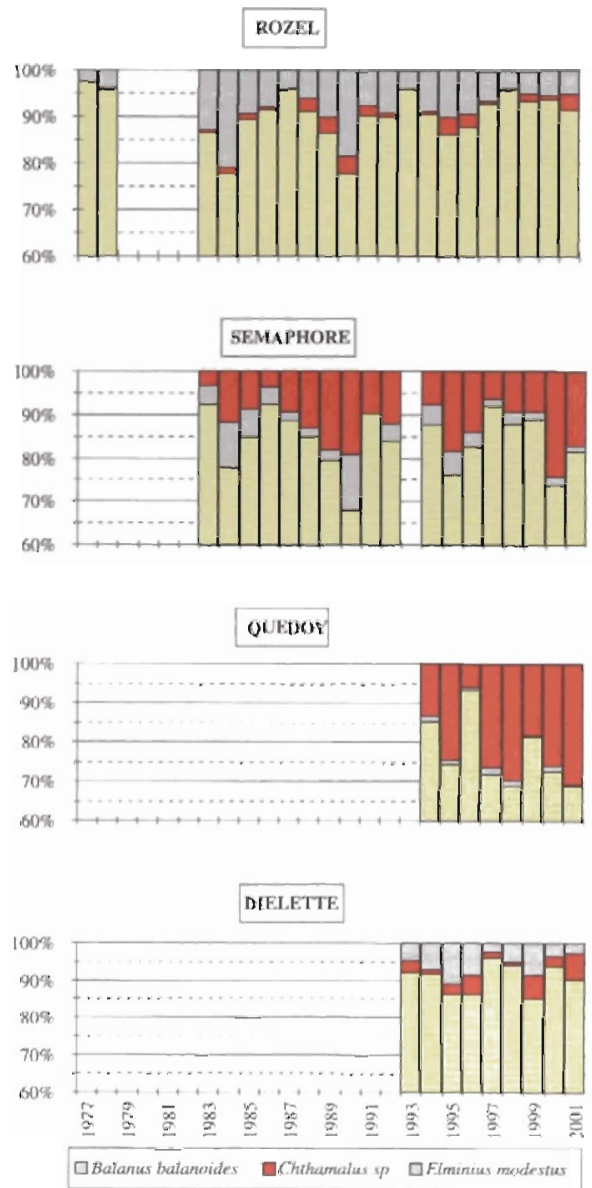

Fig. 7 - Evolution, en \%, des quatre espèces de cirripèdes (les deux espèces de Chthamales ont été regroupées au niveau du genre).

Fig. 7 - Long-term variation in population densities (N.ind. $\mathrm{m}^{2}$ ) of four intertidal barnacle species.

\section{4 ÉTUDE DE RECOLONISATION DES SUBSTRATS DURS INTERTIDAUX PAR LES CRUSTACÉS CIRRIPĖDES}

Le recrutement printanier (entre début mars et la mi-juin) représente, 
selon les années, de $77 \%$ à $99,6 \%$ du phénomène annuel, à l'exception toutefois du printemps 1990 où il n'atteint que $48 \%$ à la pointe du Rozel et $61 \%$ au cap de Flamanville. II concerne exclusivement Balanus balanoides.

Le recrutement estival (de la mi-juin à la mi-septembre), beaucoup plus faible $(2 \%$ en moyenne du recrutement annuel; de 1 à 50 individus par $75 \mathrm{~cm}^{2}$ ), concerne en priorité les trois autres espèces.

La recolonisation observée entre septembre et mars suivant $(2 \%$; de 1 à 90 individus par $75 \mathrm{~cm}^{2}$ ) n'est en fait que la fin du recrutement estival.

Le cycle de ces recrutements, décrit lors des études de projet, est confirmé depuis 1983. Les séries à long terme ainsi acquises devaient permettre de vérifier l'hypothèse comme quoi les rejets d'eau échauffée de la centrale pourraient avoir un effet sur le recrutement de ces crustacés. Ainsi, l'espèce dominante, à preferendum thermique froid, devrait voir son importance diminuée localement, suite à un éventuel affaiblissement des recrutements printaniers.

Plusieurs recrutements printaniers sont à signaler :

- Le recrutement de 1983 (612 ind. par $75 \mathrm{~cm}^{2}$ ) est quantitativement six fois supérieur à celui de 1978 (98 ind. par $75 \mathrm{~cm}^{2}$ ) alors que celui de 1984 (103ind. par $75 \mathrm{~cm}^{2}$ ) est quasi identique à ce dernier.

- Les recrutements de 1986 (2725 ind. par $75 \mathrm{~cm}^{2}$; repéré par une flèche bleue à la figure 8) et 1987 présentent des niveaux exceptionnellement élevés, alors que

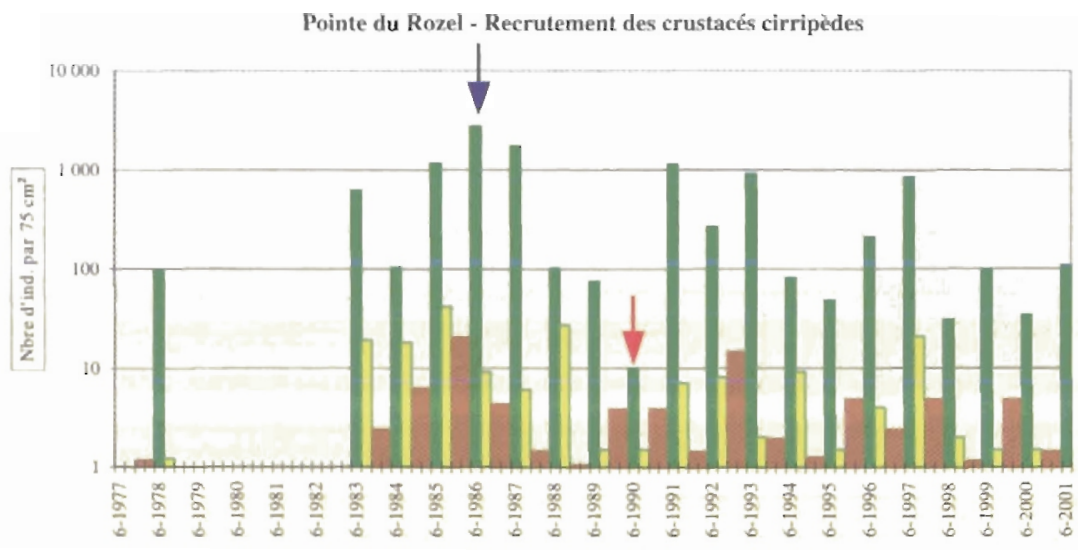

Fig. 8 - Recrutements printanier (histogramme vert), estival (jaune) et automno-hivernal (marron) des cirripèdes. Les flèches indiquent les recrutements ie plus fort (flèche bleue) et le plus faible (flèche rouge).

Fig. 8 - Recruitments of intertidal barnacles : spring (green), summer (yellow) and autumn-winter (brown). The blue arrow indicates the more important recruitment; the red arrow, the smaller. 
les deux tranches de la centrale sont progressivement mises en service. Etablir un lien de cause à effet, tentant dans le cadre d'impact des rejets de la centrale, est contredit par la biologie boréoarctique de l'espèce.

- A l'opposé, le recrutement de 1990 (figure 8 , flèche rouge) est le plus faible (10ind. par $75 \mathrm{~cm}^{2}$ ) observé sur ce site.

La ponte planctonique chez l'espèce dominante Balanus balanoides étant hivernale, nous nous sommes intéressés aux données météorologiques hivernales, principalement la température de l'air, enregistrées au sémaphore d'Auderville (au nord de La Hague). L'observation de la température moyenne mensuelle la plus froide pour chaque année permet de noter que, depuis 1977, la valeur minimale la plus basse est enregistrée en février $1986\left(2,20^{\circ} \mathrm{C}\right)$; la plus clémente en janvier $1990\left(8,90^{\circ} \mathrm{C}\right)$.

Le même constat est réalisé sur la température de l'eau de mer enregistrée quotidiennement depuis 1986 dans le canal d'amenée de la centrale de Flamanville par EDF.

L'expression des moyennes mensuelles des températures de l'eau de mer à Flamanville en fonction de celles de l'air au nord de La Hague montre une corrélation significative entre ces deux éléments (fig. 9). Les variations naturelles (échauffement printano-estival; refroidissement automno-hivernal) de la température de l'eau suivent cependant celle de l'air avec un léger retard dans le temps dû à la plus grande inertie de la masse d'eau par rapport à l'air.

Cette corrélation permet le choix du paramètre "température de l'air" pour analyser les variations du recru-

\section{T eau = $\mathrm{f}(\mathrm{T}$ air $)$ sur période 1986-2001} (moyennes mensuelles)

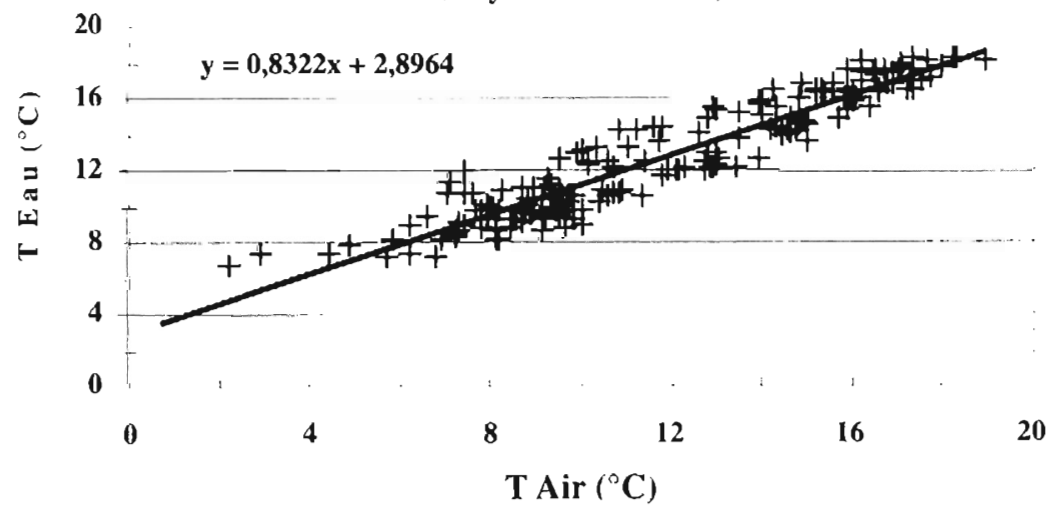

Fig. 9 - Variation de la température moyenne mensuelle de l'eau de mer en fonction de celle de l'air.

Fig. 9 - Relationship between average air temperature and sea-surface temperature for sixteen years. 
tement d'espèces animales marines, a priori plus sensibles au paramètre “température de l'eau». Ce choix permet également l'accès à une série chronologique de données plus longue (la température de l'eau n'est en effet mesurée quotidiennement que depuis 1986).

La relation entre l'importance numérique du recrutement printanier et la rigueur de l'hiver est testée (Drévès, 1995).

Pour chaque année, le recrutement printanier des crustacés cirripèdes est exprimé en fonction de la température mensuelle minimale ( $\mathrm{Ta}$ bleau I), ce mois variant entre janvier et février selon les années. Sur la période 1983-2002, l'écart thermique entre les mois hivernaux le plus froid et le plus clément est de $6,5^{\circ} \mathrm{C}$ au niveau de la température de l'air, $3,08^{\circ} \mathrm{C}$ au niveau de celle de l'eau de mer.

Ce traitement met en évidence une importance numérique de ce recrutement printanier proportionnelle à la rigueur de l'hiver (fig. 10), plus précisément une relation exponentielle négative avec la température.

Ainsi, les recrutements élevés notés pour les années 1986 et 1987 s'expliquent par des températures hivernales basses, tant au niveau de l'air $\left(2,2^{\circ} \mathrm{C}\right.$ en février $1986 ; 2,9^{\circ} \mathrm{C}$ en janvier 1987) que de l'eau de mer $\left(6,8^{\circ} \mathrm{C}\right.$ en février $1986 ; 7,21^{\circ} \mathrm{C}$ en février 1987).

A l'opposé, le faible recrutement enregistré en 1990 trouve son explication dans la clémence des tempé-

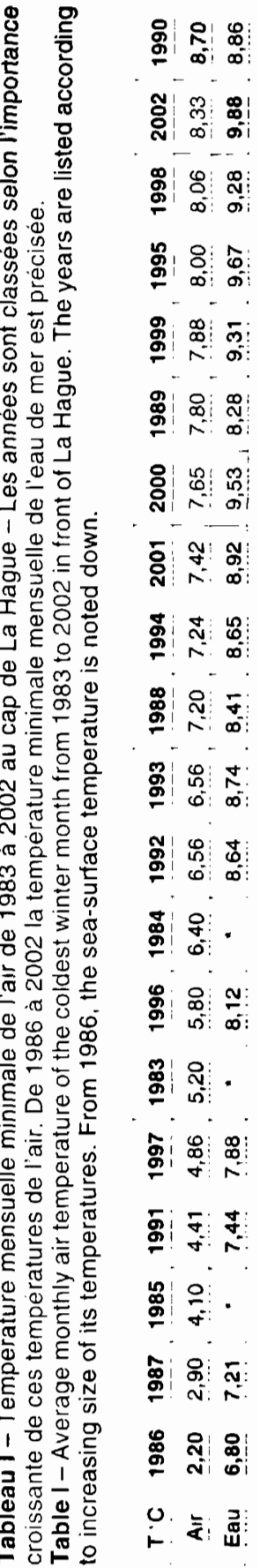



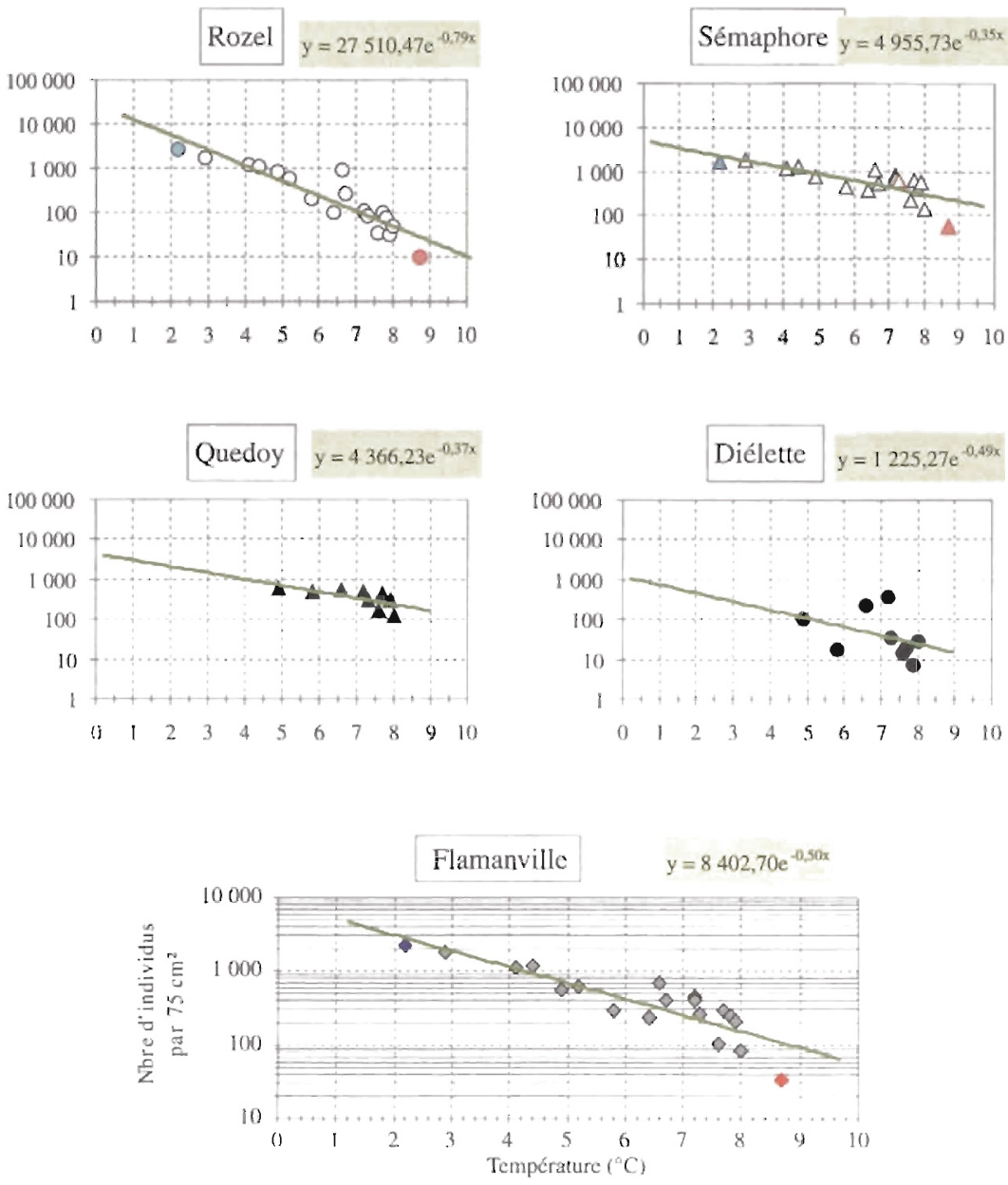

Fig. 10 - Recrutement printanier des crustacés cirripèdes aux abords de Flamanville (en nombre d'individus par $75 \mathrm{~cm}^{2}$ ) exprimé en fonction de la température mensuelle moyenne de l'air la plus froide de l'annèe (en ${ }^{\circ} \mathrm{C}$ ).

Fig. 10 - Relationship between the coldest average monthly air temperature and the numerical importance of the spring recruitment of barnacles in front of Flamanville.

ratures hivernales, $8,7^{\circ} \mathrm{C}$ pour l'air en janvier, $8,86^{\circ} \mathrm{C}$ pour l'eau en février.

Notons, pour de mêmes écarts de température, des variations plus importantes du recrutement printanier au Rozel, pointe où le peuplement des cirripèdes est le mieux représenté et le plus étendu.

La relation ainsi établie permet d'envisager, au regard de la clémence de l'hiver $2002\left(8,33^{\circ} \mathrm{C}\right.$ en janvier), un recrutement faible pour cette 
année, analogue à celui enregistré en 1990.

Sur la période 1983-2002, l'importance de la rigueur de l'hiver permet ainsi, à elle seule, d'expliquer les variations importantes des recrutements printaniers des crustacés cirripèdes intertidaux du nord-ouest Cotentin. Ces variations, d'un facteur avoisinant 300 entre les années extrêmes, démontrent l'importance des impacts météorologiques sur la biologie de certains peuplements marins. Les impacts des rejets de la centrale de Flamanville, s'ils existent sur ces espèces, ne sont pas, de ce fait, détectables.

\section{BIBLIOGRAPHIE}

Drévès L., 1995 - Recrutement printanier des cirripèdes Balanus balanoides en fonction de la rigueur de l'hiver sur la côte ouest du Cotentin (Manche-ouest, France). In: Les changements à long terme dans les écosystèmes marins méthodes d'analyse, études de cas et comparaisons inter-sites. Bachelet $G$. \& Castel J. (Eds). Colloque International, Arcachon, 1-3 février 1995, Livre des résumés.

Drévès L. et al., 2002 - Surveillance écologique et halieutique du site de Flamanville, année 2001, rapport scientifique annuel. Rapport Ifremer RST.DEL/SR/02.04, mars 2002, 154 p.

Guillaumont B. \& Hamon D., 1981 - Les études de populations zoobenthiques dans le cadre d'un état de référence sur un site de centrale thermonucléaire: Problèmes de choix. Exemples de réalisation. $2^{\phi s}$ journées de la thermo-écologie, Influence des rejets thermiques sur le milieu vivant en mer et en estuaire, Ed. EDF, 141-154.

Southward A.J., 1976 - On the taxonomic status and distribution of Chthamalus stellatus (Cirripedia) in the North-East Atlantic region : with a key to the common intertidal Barnacles of Britain. J. Mar. Biol. Ass. U.K., 56 : 1007-1028. 